\title{
Prototipagem de banco de dados: o uso da teoria da classificação facetada na modelagem de dados
}

\author{
Márcio Bezerra da Silva \\ Universidade de Brasília, Faculdade de Ciência da Informação, Brasília, DF, Brasil \\ marciobdsilva@unb.br
}

Dulce Amélia de Brito Neves

Universidade Federal da Paraíba, Departamento de Ciência da Informação, João Pessoa, PB, Brasil

damelia1@gmail.com

Resumo: Apresenta a inserção da Teoria da Classificação Facetada (TCF) na prototipagem de Banco de Dados (BD) a partir da modelagem de dados, mais especificamente nas etapas de programação: lógica e física. Discute modelagem de dados em BD como técnica que deverá representar a realidade do ambiente que o sistema será inserido. Objetiva-se, de forma maior, a partir de um percurso metodológico formado pela pesquisa aplicada, exploratória e bibliográfica, de abordagem qualitativa, apresentar a aplicação da classificação facetada para organização do conhecimento, visando à recuperação da informação em BD; e de forma específica, investigar a possibilidade de aplicabilidade da classificação facetada com a modelagem de dados em ambientes digitais e expor um panorama de uso do protótipo de BD. Alcança, como resultados da pesquisa, as contribuições da TCF na modelagem lógica e física, especificamente nos métodos de formação de assunto de Ranganathan, dissecação e desnudação, feito na modelagem conceitual (outro trabalho); e na modelagem física, ou seja, na escolha de recursos de software que, em seguida, a confeccionou os módulos do sistema em Cadastro (catalogação), Classificação e Pesquisa (busca); além de contribuições da TCF na facetação ilimitada e filtragem nas ocorrências de buscas, tornando as pesquisas mais precisas. Conclui-se que os subsídios para o desenvolvimento do Sistema Facetado foi a TCF na modelagem de dados em BD, e mesmo com os resultados satisfatórios, a grande questão encontra-se na ausência de modelos e/ou estudos empíricos que apresentem metodologias e testes, nos oferecendo como ponto de partida a imaginação e conhecimento profissional em BD. Finaliza almejando o desejo de outros estudos, outros modelos, os quais poderão nos ajudar a ampliar esta discussão para ambientes mais gerais, abrangentes, como é o caso da Web.

Palavras-chave: Banco de dados; Classificação Facetada de Ranganathan; modelagem de dados; modelagem física; modelagem lógica.

Prototipos de base de datos: el uso de la teoría de la clasificación facetada en el modelado de datos

Resumen: Presenta la inserción de la Teoría de la Clasificación Facetadas (TCF) en la creación de prototipos de base de datos (BD) desde el modelado de datos, especialmente en los pasos de programación: lógica y física. Discute BD el modelado de datos como una técnica que debe representar la realidad ambiental que se insertará el sistema. El objetivo es, en cierto modo mayor, de un curso metodológico formada por investigación aplicada, exploratoria y bibliográfica, enfoque cualitativo, presentar la solicitud de clasificación facetada para la organización del conocimiento, centrándose en la recuperación de la información en la BD; y en concreto, investigar la posibilidad de aplicación de la clasificación facetada con el modelado de datos en entornos digitales y exponer un panorama de utilizar de lo prototipo de BD. Alcanza como resultados de la investigación, las contribuciones de TCF en el modelado lógica y física, específicamente en los métodos de formación del asuntos del Ranganathan, disección y denudación, hicieron el modelado conceptual (otros trabajos); en el modelado de la física, es 
decir, la elección de las características del software, que luego los módulos del sistema confeccionó en catastro (catalogación), clasificación y búsqueda; además de las contribuciones de TCF en el filtrado ilimitado y facetado en casos de búsquedas, por lo que es la investigación más precisa. Se concluye que las subvenciones para el desarrollo del sistema de tallado fue el TCF en modelado de BD, e incluso con resultados satisfactorios, la gran pregunta es la ausencia de modelos y/o estudios empíricos que presentan las metodologías y las pruebas, nos ofrece como punto de partida la imaginación y el conocimiento profesional en BD. Termina anhelando el deseo de los otros estudios, otros modelos, que pueden ayudar a ampliar esta discusión entornos del integrales más amplios, abarcador, como es el caso de la Web.

Palabras clave: Banco de datos; Clasificación Facetas de Ranganathan; modelado de datos; modelado física; modelado lógica.

\section{Database prototyping: the use of theory of faceted classification in data modeling}

Abstract: Presents the insertion of Ranganathan Theory of Faceted Classification (TCF) in prototyping Database (BD) from the data modeling, specifically in programming steps: logic and physical. Discusses $\mathrm{BD}$ data modeling as a technique that should represent environmental reality that the system will be inserted. The objective is, in a greater way, from a methodological course formed by applied research, exploratory and bibliographical, of a qualitative approach, to present the application of faceted classification for knowledge organization, focusing on the recovery of information in BD; and specifically, to investigate the possibility of applicability of faceted classification with data modeling in digital environments and expose a view of using the BD prototype. Reaches as search results, the contributions of FCT in logical and physical modeling, specifically on Ranganathan methods formation of subjects, dissection and denudation, used in the conceptual modeling (other work); and physical modeling, i.e. the choice of software features that, then concocted the system modules in Register (cataloging), Classification and Search; in addition to FCT contributions in unlimited filtering and faceting in instances of searches, making it the most accurate research. It concluded that subsidies for the development of Faceted system was the TCF in modeling data to $\mathrm{BD}$, and even with satisfactory results, the big question is the absence of models and / or empirical studies that present methodologies and testing, offering us as a starting point the imagination and professional knowledge in BD. Ends craving the desire of other studies, other models, which can help us broaden this discussion to broader, comprehensive environments, as is the case of the Web.

Keywords: Faceted Classification; Data Modeling; Logic Modeling; Physical Modeling. 


\section{Introdução}

A cada instante, o homem procura organizar o que está a sua volta, segundo alguma forma de classificação, por meio de critérios, características, que o permitirão enxergar o mundo e se inserir em grupos. Para tanto, a informação será o elemento condutor da realização de tais ações, formada por dados que necessitam de organização, estruturação e representação, conforme signos e símbolos. Para Silva e Neves (2011, p. 540, grifo dos autores), "mesmo estando elas disponíveis em grande quantidade, o ser humano não capta todas as informações que o cercam; seleciona apenas o que considera necessário, permitindo assim a criação do que chamamos conhecimento".

$\mathrm{Na}$ prerrogativa apresentada, estudos são realizados para aprimorar e/ou criar instrumentos de organização do conhecimento, e que estes devem acompanhar as mudanças impostas pelo atual fluxo de informação, envolto de adventos tecnológicos. Com isso, destacamos a Ciência da Informação $(\mathrm{Cl})$, considerada uma área de estudo interdisciplinar. Justamente por tal característica, ou seja, pela presença de muitas áreas do conhecimento estarem relacionadas à $\mathrm{Cl}$, sobressaímos a Biblioteconomia na perspectiva da classificação bibliográfica; e a Ciência da Computação (CC), no desenvolvimento e implementação de Banco de Dados (BD). Ambas os campos de estudos nos ofereceram subsídios na busca por soluções aos problemas de uma determinada Instituição ${ }^{1}$, mais especificamente em seu Setor Pedagógico, quanto à inexistência ou má organização, tanto dos mínimos materiais físicos, quanto dos criados digitalmente.

Compreendida a importância da organização nesse processo, criamos um BD em solicitação a Direção da Instituição, com o objetivo de organizar e recuperar as informações do respectivo setor. Neste certame, no âmbito dos estudos sobre classificação bibliográfica, escolhemos a Teoria da Classificação Facetada (TCF) do indiano Shialy Rammarita Ranganathan (1897-1972), onde o sistema adotado foi incorporado ao protótipo de BD, através da modelagem de dados da CC. Inicialmente foi realizado um estudo teórico sobre o uso da TCF em BD para determinar os elementos básicos e etapas para a construção do sistema ${ }^{2}$. Em seguida ocorreu a

\footnotetext{
${ }^{1}$ Rotularemos, ao longo do trabalho, o campo da pesquisa de Instituição X.

2 SILVA, M. B. da; NEVES, D. A. B. Estudo sobre o uso da teoria da classificação facetada em banco de dados. In: ENCONTRO NACIONAL DE PESQUISA EM CIÊNCIA DA INFORMAÇÃO, 11, 2010, Rio de Janeiro. Anais eletrônicos... Rio de Janeiro: CPRM - UNIRIO, 2010. 20p. Disponível em: <http://congresso.ibict.br/index.php/xi/enancibXI/paper/view/283>. Acesso em: 10 ago. 2014. RICI: R.Ibero-amer. Ci. Inf., ISSN 1983-5213, Brasília, v. 9, n. 1, p. 242-257, jan./jun.2016. 
fase inicial da modelagem de dados, ou seja, a etapa conceitual ${ }^{3}$, momento onde foram definidos os instrumentos para representar a realidade da Instituição, fazendo uso de campos do Código de Catalogação Anglo-Americano 2 (AACR2) para a descrição dos materiais; indexação para a escolha dos termos representativos; formação de assuntos da TCF; e definição de facetadas conforme a mnemônica Personalidade, Matéria, Energia, Espaço e Tempo (PMEST). Por fim, na atuação edição, tivemos à pretensão de apresentar as outras etapas da modelagem de dados (modelagens lógica e física), nos permitindo observar o protótipo de BD em funcionamento.

A referida observação calcou-se na contínua busca em responder a seguinte indagação: a classificação facetada pode contribuir para organização do conhecimento registrado em BD, visando à recuperação da informação com maior valor agregado?

A partir do contexto apresentado, baseado em um percurso metodológico formado pelas técnicas de pesquisa aplicada, exploratória e bibliográfica, de abordagem de coleta de dados qualitativa, objetivou-se, de maneira geral, apresentar a aplicação da classificação facetada para organização do conhecimento, visando à recuperação da informação em BD. De forma específica, buscou-se investigar a possibilidade de aplicabilidade da classificação facetada com a modelagem de dados em ambientes digitais e expor um panorama de uso do protótipo de BD.

\section{Modelagem de Dados em Banco de Dados}

O BD deve ser criado respeitando a realidade do ambiente que será inserido. Para tanto, fatores como os usuários, tipos de informação e recursos de hardware e software adotados etc. devem ser levados em consideração. Em outras palavras, será “[...] um modo de estruturar logicamente as informações" (FURTADO; SANTOS, 1980, p. 37). A essa estruturação damos o nome de modelagem de dados, compreendido por Elmasri e Navathe (1994) como um conjunto de conceitos usados para descrever a estrutura do BD e certas restrições que o banco deve garantir. Conforme Miranda (2005), a estruturação por modelagem permite ao usuário recuperar dados de forma rápida e eficiente, cujo objetivo é incluir dados em uma estrutura que possibilite transformar os dados originais em vários tipos de saídas como formulários, relatórios, etiquetas ou gráficos.

\footnotetext{
${ }^{3}$ SILVA, M. B. da; NEVES, D. A. B. A aplicação da teoria da classificação facetada em banco de dados através da modelagem conceitual. In: ENCONTRO NACIONAL DE PESQUISA EM CIÊNCIA DA INFORMAÇÃO, 11, 2011, Brasília. Anais eletrônicos... Brasília: Thesaurus, 2011. 20p. 1 CD-ROM. 
Um dos pontos críticos da modelagem de dados está além do conhecimento técnico, como enfatizado anteriormente. Estamos nos referindo ao conhecimento sobre a realidade em que o BD será construído, buscando solucionar algum tipo de problema. Neste sentido, Machado e Abreu (1996, p. 22) defendem a preocupação com a realidade ao expor que:

Para registramos as necessidades de informação de uma realidade, precisamos fazer uso de um modelo, ou seja, algo que nos mostre como as informações estão relacionadas (fatos). E com base no modelo criado, os analistas podem interagir com os usuários validando seus objetivos e metas, permitindo a construção de um sistema de informações cada vez mais próximo de realidade.

Aliados à preocupação com a realidade, na modelagem de dados existe uma quantidade de abstração de informações que varia conforme o tipo de usuário e modelo de dados escolhido. Chamado de Nível de Abstração dos Dados, esta característica representa a omissão de detalhes sobre o armazenamento das informações para alguns usuários (KORTH; SILBERSCHATZ, 1995) e classifica-se em três modelos: nível conceitual, nível lógico e nível físico.

\section{As modelagens Lógica e Física na Prototipagem do Banco de Dados}

Como já foi apresentada, a modelagem de dados é composta por três etapas de abstração. Na primeira, a conceitual, a maior contribuição da $\mathrm{Cl}$ foi a TCF, apresentando o nome sugestivo de Sistema Facetado ao Sistema de Banco de Dados (SBD). Foi exatamente a flexibilidade, ou seja, a possibilidade de criar e recriar novos agrupamentos entre as facetas, sem alterar significativamente a estrutura de programação e relacionamentos das tabelas do $B D$, que propiciou a sua escolha para este trabalho.

Após a modelagem conceitual, chegamos às instruções de implementação do sistema. Na modelagem lógica, são apresentados maiores detalhes sobre o armazenamento dos dados e como serão relacionados. Conforme Heuser (2004), a modelagem lógica é o resultado ou produto da conversão de uma modelagem conceitual para um determinado tipo de banco de dados. Para Machado e Abreu (1996, p. 24), "a modelagem lógica objetiva descrever "[...] as estruturas que estarão contidas no banco de dados, de acordo com as possibilidades permitidas pela abordagem, mas sem considerar, ainda, nenhuma característica específica de um $[\ldots]$ SGBD $\left.^{4}[\ldots]\right]^{\prime}$. O resultado é um esquema lógico de dados, construído a partir de um tipo de BD.

\footnotetext{
${ }^{4}$ Sistema Gerenciador de Banco de Dados. 
Dentre os tipos, adotamos o modelo relacional, que consiste em um conjunto de tabelas, onde suas linhas representam um relacionamento entre um grupo de valores. Atualmente, muitos dos SGBD fazem uso do modelo relacional no desenvolvimento de suas aplicações. Date (2003, p. 93) complementa ao afirmar que "a base da moderna tecnologia de banco de dados é, sem dúvida, o modelo relacional".

O modelo relacional, pela sua multidimensionalidade, foi o escolhido por apresentar características semelhantes à TCF, especialmente pela inserção de novos dados sem alterações significativas ao modelo estruturado. Neste sentido, foi possível inserir facetas e relacioná-las entre as tabelas do Sistema Facetado.

A representação da modelagem conceitual em lógica foi criada no software Microsoft (MS) Office Visio, versão 2007. Apresentando formas predefinidas, modelos inteligentes e exemplos, o MS Office Visio oferece uma grande variedade de opções para atender às necessidades de diagramação em tecnologia da informação (TI), negócios, gerenciamento de processos e muito mais. O Visio possui uma categoria específica para BD e, dentre as suas opções, usamos o Diagrama de Modelo de Banco de Dados, conforme ilustrado na figura 1.

Figura 1: Diagrama de Modelo de BD.

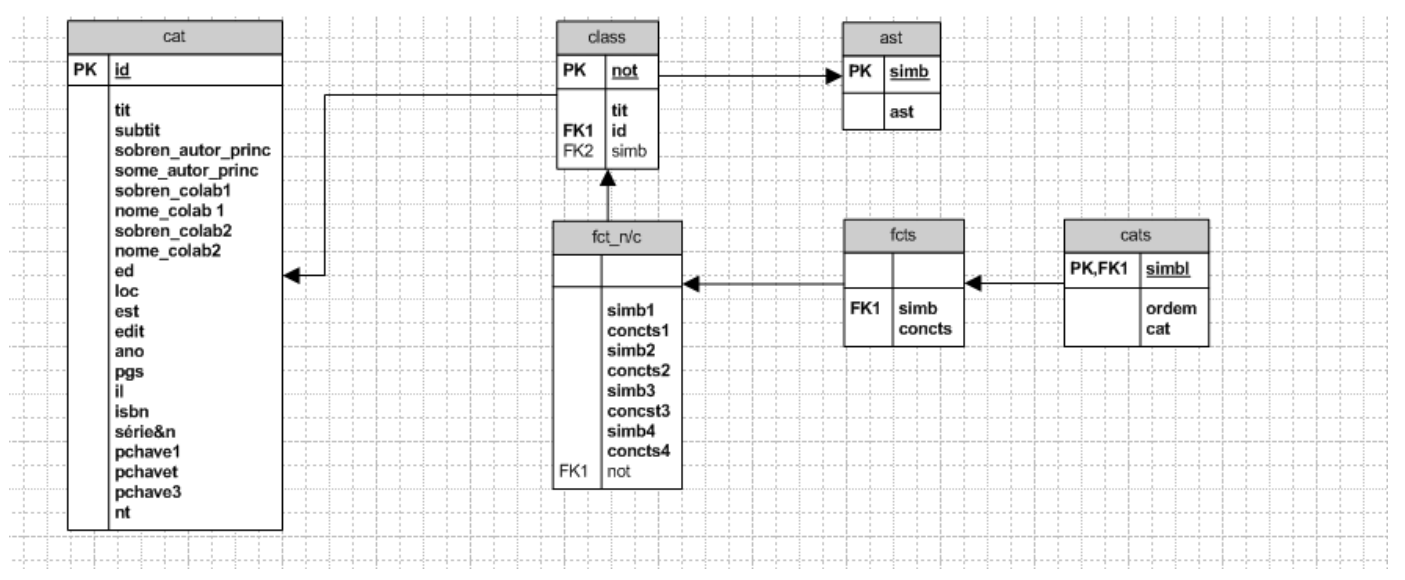

Fonte: MS Visio 2007, Sistema Facetado (2011-2013).

Conforme pode ser observado na figura 1, a proposta de implementação do Sistema Facetado está organizada em diagramas, que representam as respectivas tabelas (entidades) do sistema, que por sua vez apresentam os dados e seus atributos, e, por fim, seus relacionamentos.

A construção dos diagramas foi idealizada respeitando as características coletadas na modelagem conceitual, ou seja, conforme a atuação da TCF na formação dos assuntos, dentre os métodos Dissecação (Dissecation), o qual permitiu identificar que "Educação RICl: R.Ibero-amer. Ci. Inf., ISSN 1983-5213, Brasília, v. 9, n. 1, p. 242-257, jan./jun.2016. 
Profissionalizante em Informática" ${ }^{5}$ era o assunto básico, também rotulado pela Instituição de "Capacitação Profissional"; e Desnudação (Desnudation), para que o sistema tivesse uma classificação ilimitada, permitindo que o usuário fosse o mais específico possível.

Apesar da primeira tabela, "Catalogação - cat", ser a inicial do sistema e a que recebe as informações dos materiais, a tabela mais importante foi "Facetas_nível/ciclo - fct_n/c", pois era o elemento responsável em apresentar as facetas e disponibilizá-las à Classificação, que por sua vez está relacionada ao Cadastro, por um lado, e ao Assunto, pelo outro. Por esse motivo a referida tabela ficou no núcleo do $\mathrm{BD}$, alimentando a multidimensionalidade nos momentos de classificação e recuperação da informação.

$\mathrm{Na}$ passagem da modelagem lógica para a física, ocorreram as definições (configurações) nas "estruturas físicas de armazenamento dos dados, tais como: tamanho dos campos, índices, tipo de preenchimento destes campos, nomenclaturas, etc., projetados de acordo com os requisitos de processamento [...]" (MACHADO; ABREU, 1996, p. 25).

Esta é a etapa final do desenvolvimento do BD, preparando o sistema para ser usado pelo usuário. Nesta etapa (física), o nível de abstração é mínimo, pois a estrutura de armazenamento do BD e seus dados serão apresentados de maneira definitiva, ou seja, será disponibilizada a versão final ao usuário.

Selecionados os dados que constituirão o sistema e sua organização lógica, a próxima etapa foi escolher os recursos de software, conforme as características oferecidas pelo Sistema Gerenciador de Banco de Dados (SGBD). Neste ponto, usamos os Sistemas Operacionais (SO) MS Windows $X P^{6}$ e MS Windows $7^{7}$. Quanto ao SGBD, selecionamos o MS Access, por desenvolver aplicações sob a abordagem relacional, ou seja, adota o tipo de BD relacional em sua modelagem lógica. Além disso, foi uma solicitação da Instituição, já que possui a assinatura digital do Pacote Microsoft Office, versões 2003 e 2007. A versão utilizada para o desenvolvimento do sistema foi a 2007 e testada/atualizada na versão 2010. No que se refere às estruturas de programação, adotamos o Structured Query Language (SQL), para a realização das consultas, e o Visual Basic for Applications (VBA), para a programação mais especializada de partes do sistema, especialmente as relacionadas a classificação e a pesquisa.

O MS Access é um SGBD relacional e por isso sua programação ocorreu por meio de tabelas. No protótipo, conforme as definições da modelagem física, quanto aos recursos de

\footnotetext{
${ }^{5}$ Também chamado na Instituição de "Capacitação Profissional" ou "Capacitação Profissionalizante".

${ }^{6}$ Na primeira versão do sistema, 2011. A segunda versão foi produzida no final de 2011.

${ }^{7} \mathrm{Na}$ terceira versão do sistema, 2013. Último acesso para testes em 20/02/14. RICI: R.Ibero-amer. Ci. Inf., ISSN 1983-5213, Brasília, v. 9, n. 1, p. 242-257, jan./jun.2016.
} 
software, foram criadas seis tabelas (entidades), conforme podem ser observados na figura 2, além dos seus campos (atributos). Também foram criadas três consultas para realizarem as pesquisas no sistema: Cons_Cat (consultar o cadastro), para consultar informações cadastrais do material após pesquisa realizada; Cons_Classif (consultar a classificação), necessária para associar o material aos seus conceitos (PMEST) no momento da pesquisa; e Cons_Rec (módulo de recuperação da informação), considerado um dos objetos mais importantes do sistema, pois é a partir desta consulta que as informações serão recuperadas e filtradas a partir das facetas (PMEST). Também foram criados formulários para a interface do sistema e uso, como Menu, Categorias, Classificação, Recuperação etc., além de dois relatórios.

Figura 2: Relações entre as tabelas do Sistema Facetado.

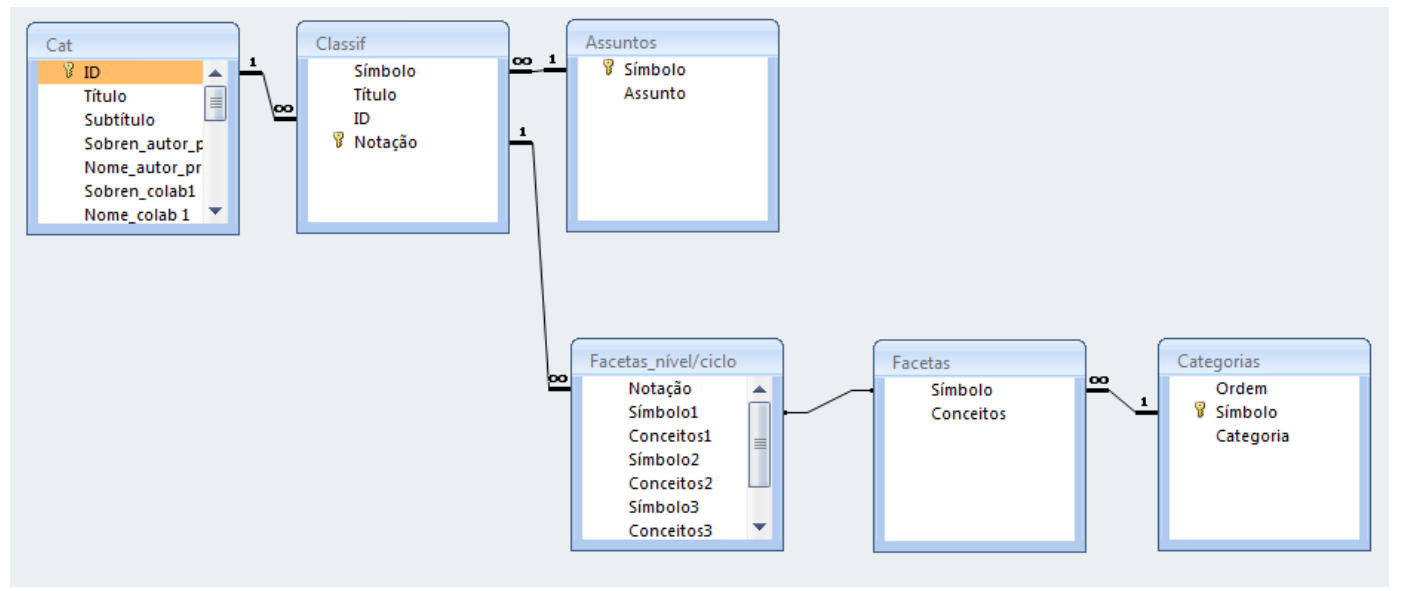

Fonte: MS Access 2010, Sistema Facetado (2011-2013). Último acesso em 2014.

As tabelas e relacionamentos apresentados são uma tradução da modelagem lógica criada na etapa anterior. Primeiramente, o material é cadastrado no sistema. Em seguida, deve ser classificado em algum assunto e receber uma notação. Na tabela classificação, ocorrem três relações, sendo a primeira listar informações do material cadastrado, a segunda listar os assuntos presentes no sistema e a terceira adotar as facetas correspondentes ao assunto determinado.

Completado este ciclo, o material está classificado e pronto para ser recuperado pelo usuário. O sistema apresenta uma tela inicial contendo um Menu (figura 3), organizado em três seções. A primeira chama-se Módulos, contendo Cadastro (catalogação), Classificação e Pesquisa. A segunda seção é formada pelos Relatórios, formado por Materiais, que listará todos os objetos cadastrados no sistema; e Conceitos, que apresentará uma lista de todos os conceitos presentes no sistema, organizados por categoria. A terceira e última seção, chamada de Informação, foi uma solicitação da Instituição para alertar os usuários quanto aos objetivos RICI: R.Ibero-amer. Ci. Inf., ISSN 1983-5213, Brasília, v. 9, n. 1, p. 242-257, jan./jun.2016. 
do sistema e que os mesmos não solicitem materiais antes de realizar consultas, para verificar a presença ou não de determinado material no acervo do Setor Pedagógico.

Figura 3: Menu Principal.

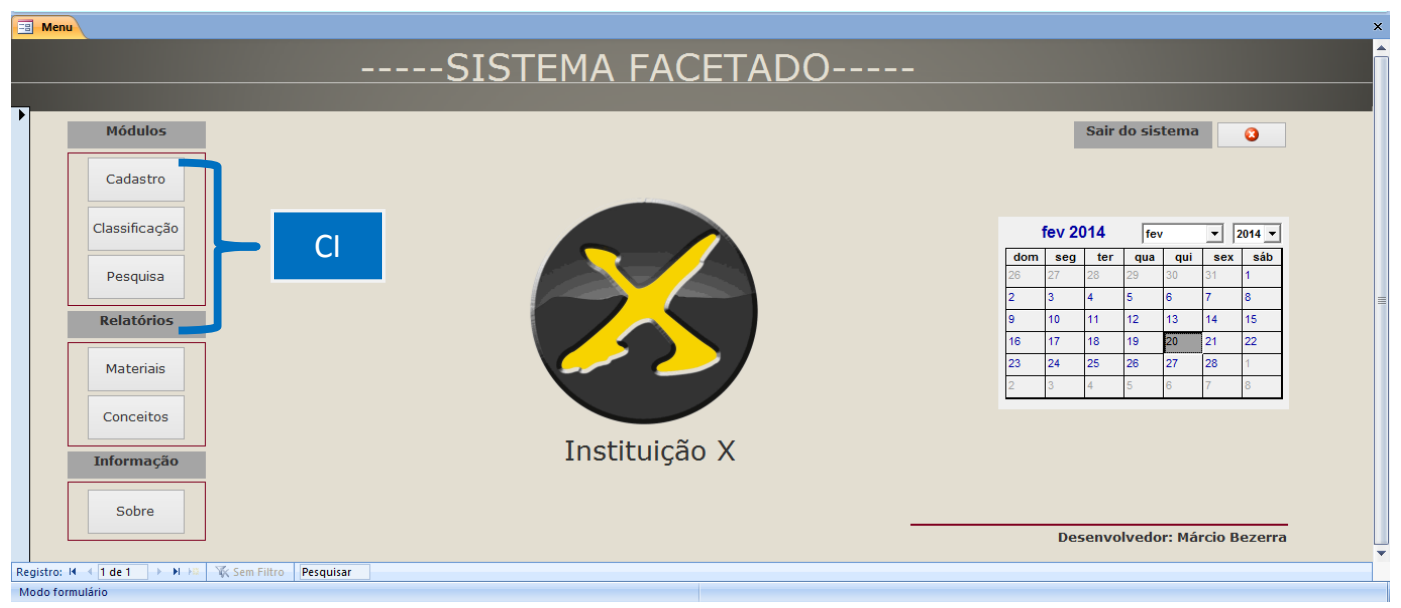

Fonte: MS Access 2010, Sistema Facetado (2011-2013). Último acesso em 2014.

Na seção Módulo, o primeiro botão remete o usuário ao Cadastro de Materiais. Esta opção atua segundo a representação descritiva da informação, ou seja, a catalogação (figura 4), mais especificamente campos básicos do AACR2. A ação e o código adotado foram subsídios da $\mathrm{Cl}$ para e elaboração deste módulo.

Figura 4: Módulo Cadastro de Materiais.

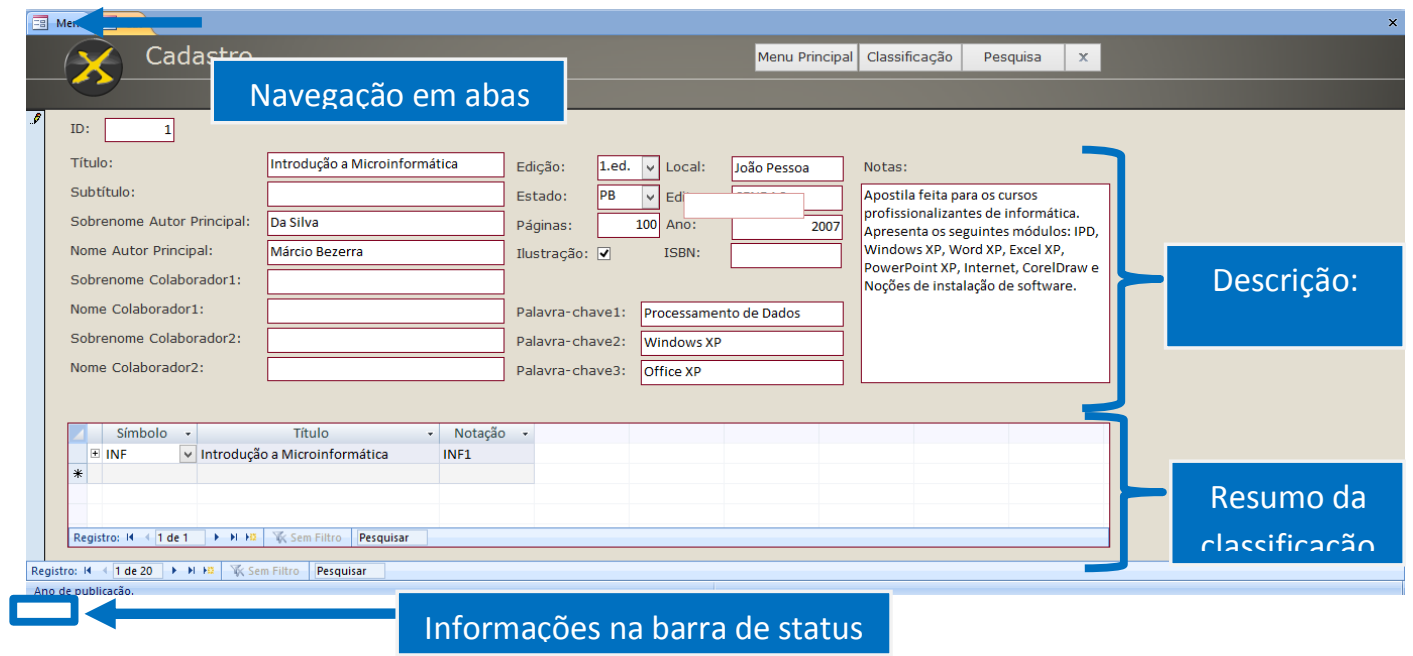

Material aberto (atual) e quantidade de materiais

Fonte: MS Access 2010, Sistema Facetado (2011-2013). Último acesso em 2014. 
Na parte inferior da tela, figura 4, temos um resumo do material classificado e sua respectiva notação ${ }^{8}$ de sistema. Além disso, na barra de status, os usuários são informados sobre o preenchimento de cada campo por meio de mensagens.

Para que as informações da notação, de determinado material, constem no módulo Cadastro de Materiais, é necessário acessar o módulo Classificação (figura 5), que representa outros subsídios da $\mathrm{Cl}$, ou seja, a TCF e a classificação. Neste módulo, o usuário seleciona o símbolo do assunto e em seguida a $\mathrm{ID}^{9}$ do material cadastrado no módulo anterior. No campo título, conforme a seleção da ID, automaticamente surge o título do material cadastrado, realizado no módulo anterior, Cadastro de Materiais. Possuindo o símbolo do assunto e o número da ID do material, é formada a sua notação (INF ${ }^{10}$ [Símbolo do Assunto] +1 [ID] = INF1). A notação também será apresentada no módulo Cadastro de Materiais, na parte Resumo da Classificação (figura 4).

Figura 5: Módulo Classificação.

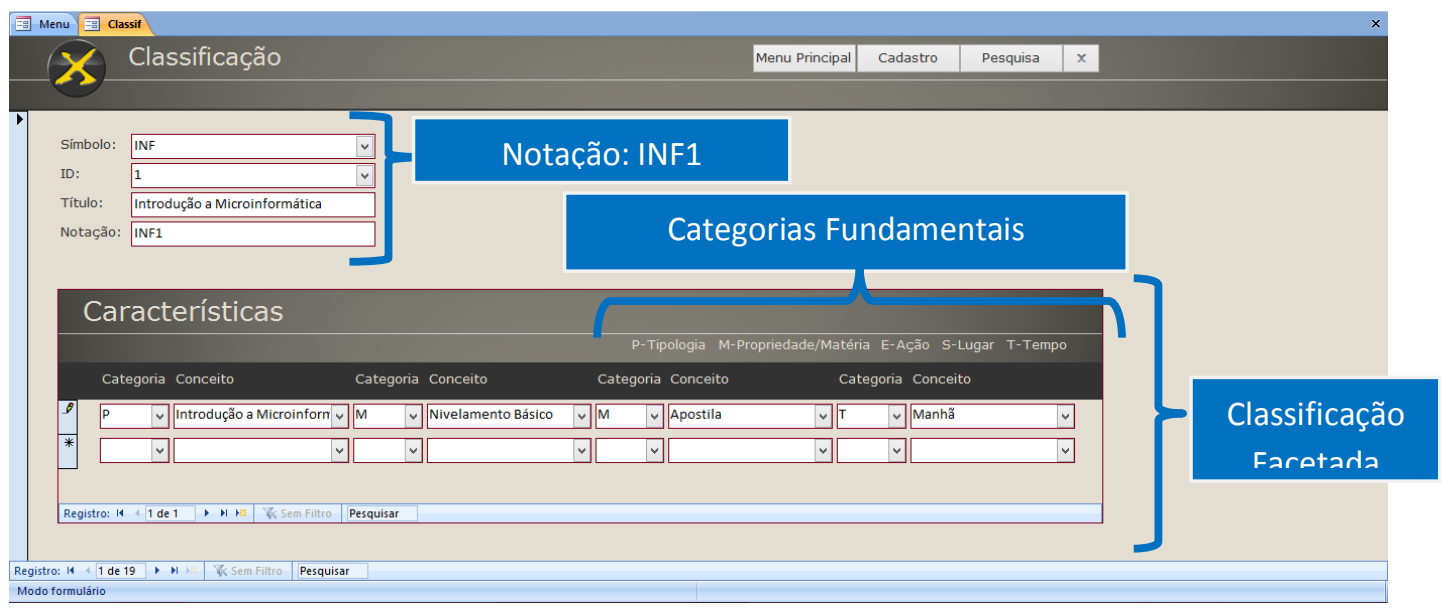

Fonte: MS Access 2010, Sistema Facetado (2011-2013). Último acesso em 2014.

Na parte Características, o usuário determinará as facetas para cada material, a ser chamado de conceitos, organizados em categorias $^{11}$, que por sua vez receberam outras denominações para representarem, de maneira mais fidedigna, a realidade do Setor Pedagógico. Esta atualização foi realizada na modelagem conceitual, a saber: "Personalidade [P] assumiu Tipologia; Matéria [M] assumiu Propriedade (qualidade) e Matéria (substância);

\footnotetext{
${ }^{8}$ Apenas para controle de ordem no sistema.

${ }^{9}$ ID: Identidade gerada pela numeração automática do sistema, conforme a entrada do material no protótipo.

${ }^{10}$ Para os testes desta pesquisa, usamos apenas os materiais de informática (INF). Outras áreas estão sendo implementadas com os seus respectivos símbolos.

${ }^{11}$ Um mapa categorial foi criado, organizado por facetas, para orientar os usuários na classificação. O mesmo foi atualizado em fevereiro do corrente ano. 
Energia [E] assumiu Ação; Espaço [S] assumiu Lugar; e Tempo [T] manteve sua rotulação" (SILVA; NEVES, 2011, p. 552). Conforme é apresentado na figura 6, verificamos que, ao selecionar de uma categoria pelo usuário, os respectivos conceitos surgem para realização da classificação facetada.

Na primeira versão, apenas a categoria [P] foi implementada e parte do conjunto [M]. $\mathrm{Na}$ última atualização foram inseridas todas as facetas da categoria [M], como também os demais conjuntos $[E],[S]$ e $[T]$, conforme proposta inicial do sistema.

Figura 6: Características dos materiais segundo categorias.

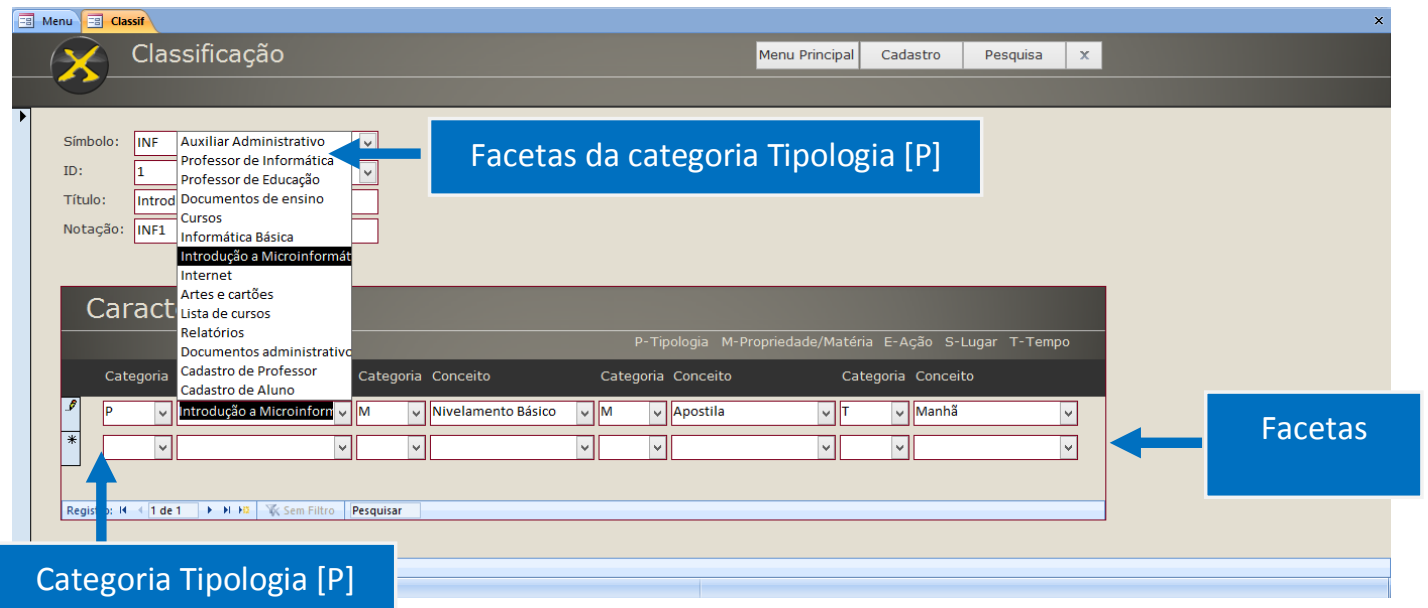

Fonte: MS Access 2010, Sistema Facetado (2011-2013). Último acesso em 2014.

O uso do PMEST, em alguns momentos, pode apresentar um assunto que necessite da presença de uma mesma categoria mais de uma vez. Quando isso ocorre, temos os Levels (níveis) e os Rounds (ciclos). Os níveis ocorrem diante da aparição de duas ou mais categorias relacionadas ao mesmo assunto principal, representados pela sigla de sua categoria e seguidos do algarismo arábico que indicará a ordem sequencial. Assim, na classificação (atualizada) "INF1[P],[M],[M2],[T]", verificamos o segundo nível da Matéria-Propriedade (M2), do título "Introdução a Microinformática" (INF1).

Os ciclos ocorrem quando uma categoria precisa reaparecer após o surgimento de outro tipo de categoria. Retornando ao título "Introdução a Microinformática" (INF1), acrescentamos um ciclo após o segundo nível da Matéria-Substância. Ao mudarmos $[T]$ por um novo $[P]$ (no final da classificação), temos "INF1[P]:[M],[M2],[2P]", ou seja, constatamos, após MatériaSubstância, o segundo ciclo de Tipologia (2P).

Conforme a classificação facetada, os materiais serão recuperados segundo o foco atribuído pelo usuário. Ambas as ações, Cadastro e Classificação, são obrigatórias para que os 
materiais sejam encontrados (apresentados) no módulo Pesquisa. Ao buscarmos pelo termo Windows ${ }^{12}$, por exemplo, o sistema apresentou a ocorrência de 10 materiais, conforme pode ser observado na figura 7.

Figura 7: Módulo Pesquisa.

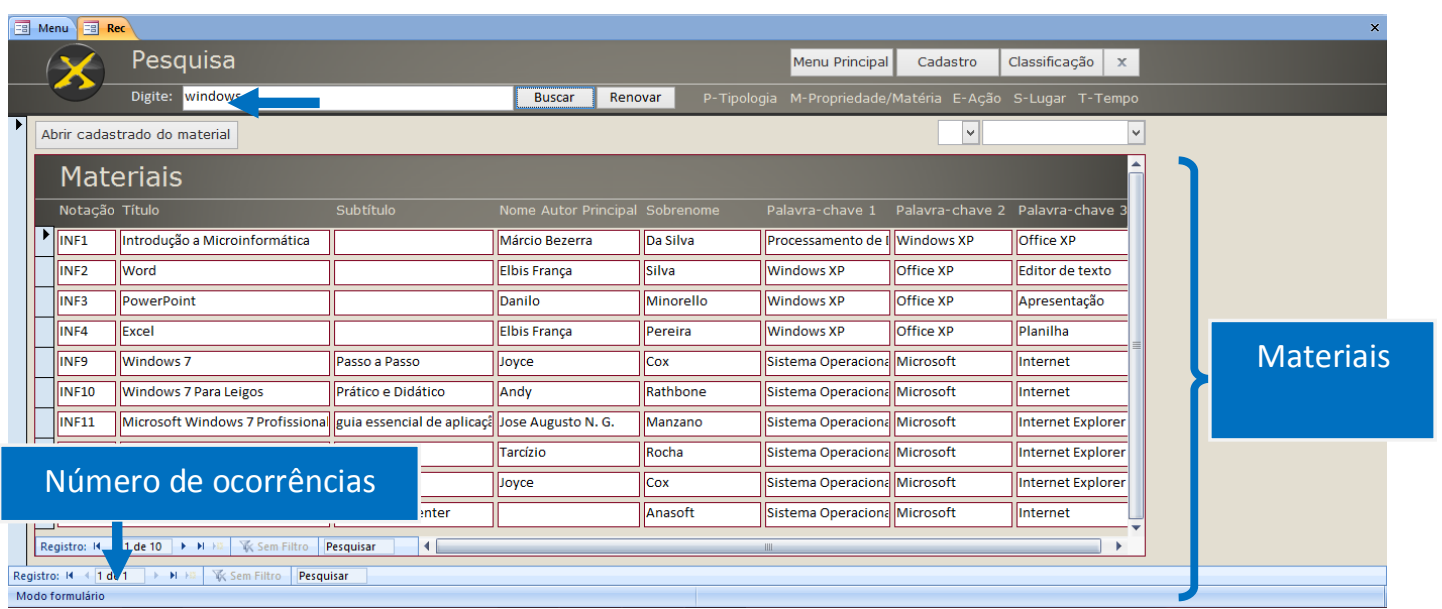

Fonte: MS Access 2010, Sistema Facetado (2011-2013). Último acesso em 2014.

Depois de realizada a busca, no item Materiais surgiram ocorrências que atenderam ao que foi digitado pelo usuário, apresentadas de maneira resumida. Para tanto, foi criado um algoritmo, chamado algoritmo_pesquisa, que fizesse, em primeiro nível, uma busca pelos campos do módulo Cadastro, considerados os mais importantes pelos usuários do sistema: Notação, Título, Subtítulo, Sobrenome Autor Principal, Nome Autor Principal, Palavra-chave1, Palavra-chave2 e Palavra-chave3.

Caso o usuário tenha o desejo de especificar as ocorrências, neste caso, por materiais que também abordem o assunto Internet, basta digitar no campo de pesquisa que ocorrerá uma filtragem no resultado anterior de busca. A partir do algoritmo_filtro_ocorrencia, ocorrendo em segundo nível e intitulado de Filtragem de Ocorrência, o resultado (figura 7) diminuiu para quatro ocorrências (figura 8).

\footnotetext{
12 Busca realizada por um usuário no último acesso (20/02/2014) como teste, após a atualização.
} 
Figura 8: Módulo Pesquisa.

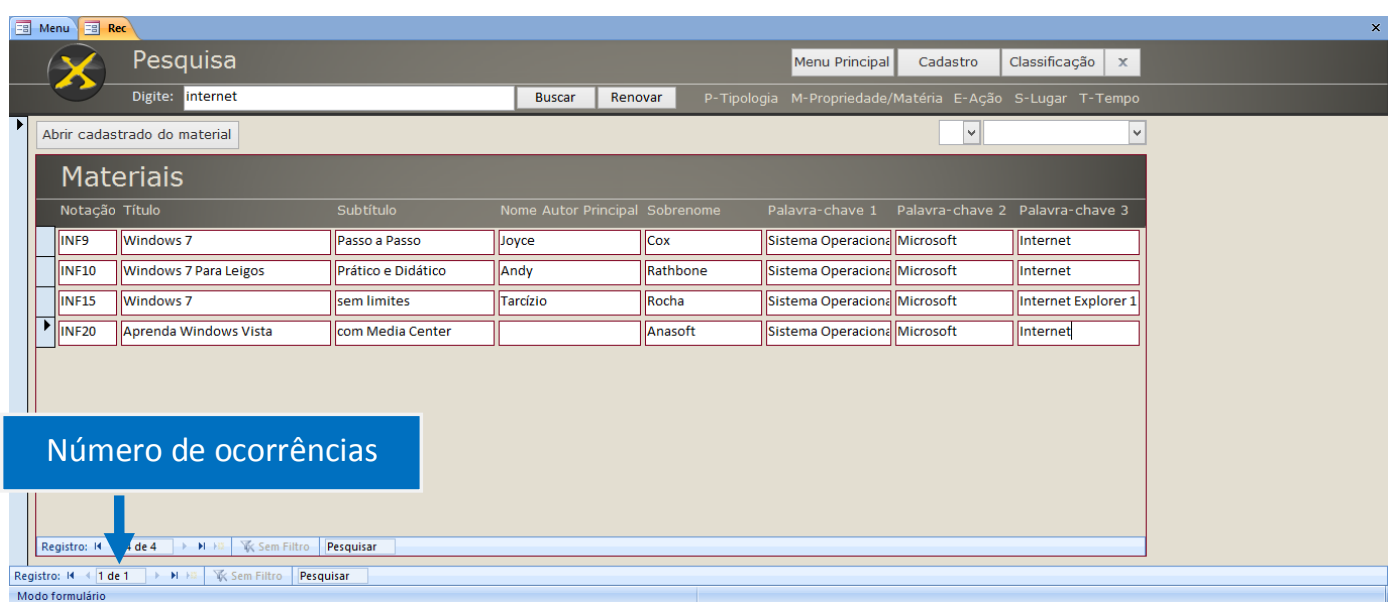

Fonte: MS Access 2010, Sistema Facetado (2011-2013). Último acesso em 2014.

Além desta filtragem, encontramos na TCF uma grande aliada, pois, ao selecionar determinada categoria e, posteriormente um conceito, o sistema filtrará o resultado da busca anterior, tornando-o ainda mais específico, conforme definido na formação de assunto Desnudação. Neste sentido, criamos o algoritmo de terceiro nível algoritmo_faceta, que por sua vez é usado quantas vezes forem necessários na busca. Exemplificando, no desejo de encontrar apenas os materiais, da busca anterior, no formato CD-ROM, quando selecionada a categoria Matéria-Substância [M] e em seguida a opção CD-ROM, tivemos apenas uma ocorrência, conforme apresentado na figura 9.

Figura 9: Busca filtrada por categoria e conceito no Módulo Pesquisa.

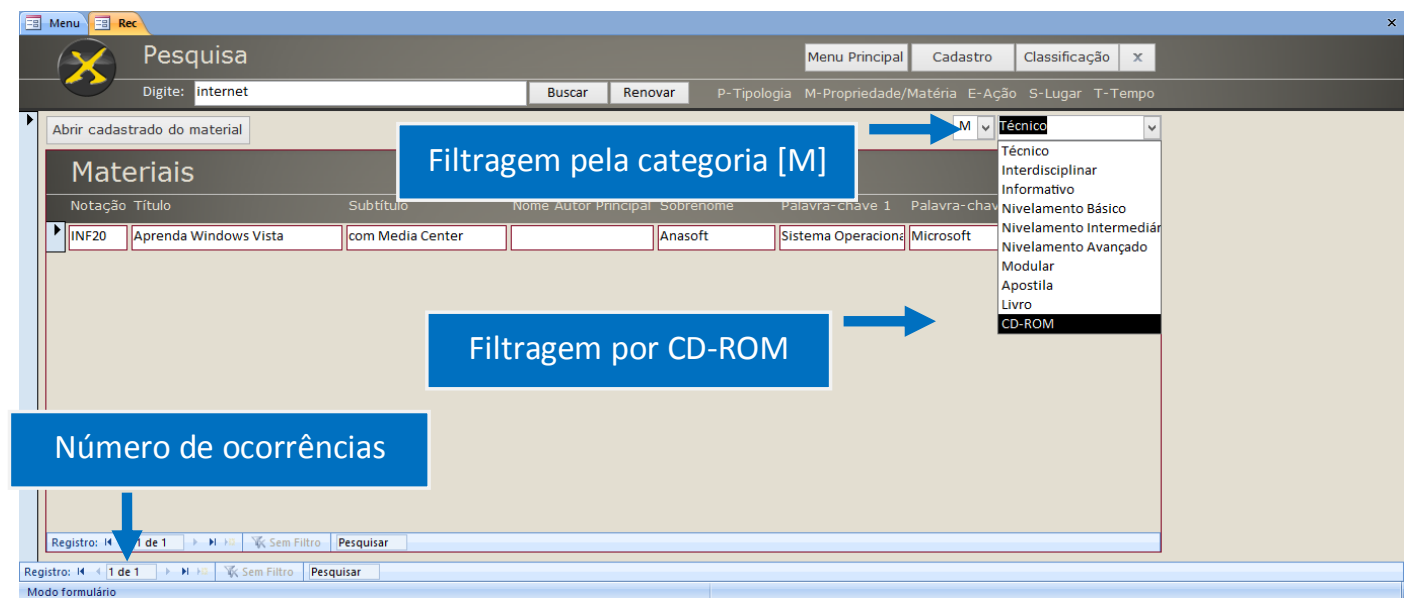

Fonte: MS Access 2010, Sistema Facetado (2011-2013). Último acesso em 2014. 
Selecionado o material, seguindo orientações da Instituição, criamos um botão chamado Abrir Cadastro de Material (figura 10) para que seja digitado o número da ID do respectivo material e, assim, ser direcionado ao módulo Cadastro.

Figura 10: Botão para abrir o cadastro do material selecionado.

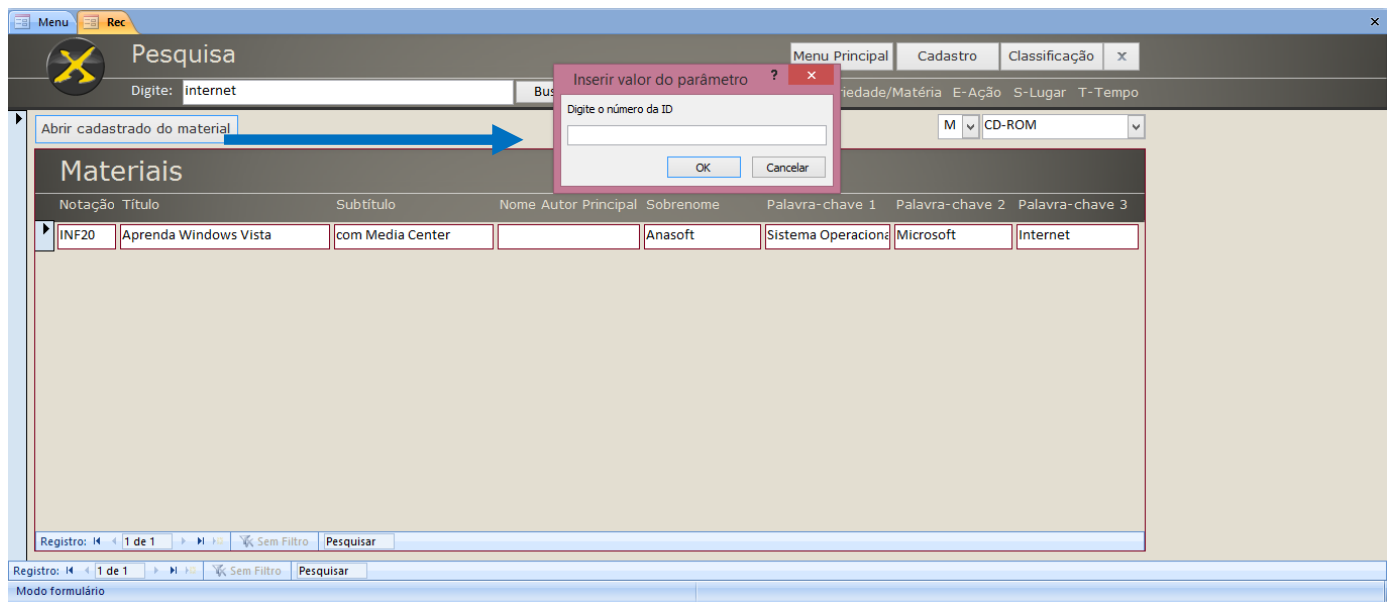

Fonte: MS Access 2010, Sistema Facetado (2011-2013). Último acesso em 2014.

Após conferir se realmente o material selecionado atenderá suas necessidades, o usuário acessará o material no formato digital ou dirigir-se-á a estante, onde o material encontra-se organizado. No caso da última busca exemplificada, deve-se digitar a ID "20", correspondente a notação INF20.

A construção do Sistema Facetado foi inspirada por modelos de Sistemas de Automação em Biblioteca (SAB). Naturalmente, ocorre à associação a classificação de livros, tarefa esta especializada dos bibliotecários. No contexto apresentado, para Litton $(1975$, p. 1), "[...] devemos recordar que o homem está constantemente ordenando coisas e que a faculdade de classificar é um dos processos mentais essenciais". Diante disso, assim como é defendido pelo autor, entendemos que a classificação facetada vai além de sua utilização em livros, ordenando e classificando os mais variados suportes informacionais, especialmente os digitais.

\section{Considerações Finais}

Ao longo do presente trabalho, resolvemos discutir o que se encontrava por trás da interface do sistema, ou seja, a organização/classificação, para fins de recuperação. Para tanto, a união entre a $\mathrm{Cl}$ e $\mathrm{CC}$, ambas interdisciplinares, nos ofereceram os elementos para a construção do sistema. 
As contribuições oferecidas por ambas as áreas foram inúmeras. No caso deste trabalho, buscamos adotar estudos da $\mathrm{Cl}$ numa aplicação da CC, com o objetivo de catalogar os materiais do Setor Pedagógico da Instituição, classificá-los e, por fim, recuperá-los. Ainda nas práticas da $\mathrm{Cl}$, enraizadas na Biblioteconomia, adotamos a TCF em um ambiente digital, neste caso, em um BD. Após estudos, muitas foram às semelhanças encontradas. Dentre elas, percebemos que, assim como na classificação facetada, os sistemas computacionais por modelagem de dados, em BD, também objetivam a estruturação do conhecimento, através da organização de seus conceitos e da criação de relacionamentos entre eles, permitindo o mapeamento de uma área de assunto e a inclusão de novos conceitos - sem que isto altere a estrutura do sistema.

Depois dos estudos e o desenvolvimento do Sistema Facetado, constatamos que a análise de determinado assunto por facetas significou que o mesmo era visualizado pelas manifestações das características ou facetas atribuídas a ele, o que chamamos de conceitos. Estas manifestações são importantes, pois o Sistema Facetado tornou-se multidimensional e ilimitado, representadas pelas facetas e organizadas por categorias.

Outro ponto forte foi na etapa de recuperação da informação. Além da forma tradicional de busca por digitação, acrescentamos uma Filtragem de Ocorrência dentro da própria busca (preliminar), permitindo que os usuários refinassem a sua busca digitando outros termos. Vendo os benefícios desta técnica, adotamos também uma filtragem por facetas. Neste sentido, o usuário escolheria uma categoria fundamental e uma de suas facetas para realizar a filtragem, quantas vezes fossem necessárias. Com isso, a ideia de Ranganathan, de apresentar características aos assuntos, permitindo benefícios à recuperação, foi concretizada. De fato, a TCF permitiu tornar o assunto mais específico e, com isso, uma busca mais precisa.

A grande questão deste trabalho encontrou-se na ausência de modelos e/ou estudos empíricos que apresentassem metodologias e testes. O desenvolvimento do protótipo de BD partiu da imaginação e conhecimento profissional em BD. Conforme leituras e virtualização (mental) de como a TCF poderia ser inserida no sistema, escolhemos a programação em BD Relacional, e foi justamente este método que nos permitiu a concretização do presente trabalho.

Por fim, o Sistema Facetado, no final do ano de 2013, recebeu a última atualização e os usuários inseriram materiais de outros cursos, como também o desenvolvedor implementou todas as categorias e seus conceitos conforme a proposta inicial. Após a segunda atualização, pelo sucesso da proposta, a Instituição adquiriu os direitos legais. A partir destes 
acontecimentos, esperamos novos estudos, novas propostas, com fins a melhorias nas formas de recuperação de informação nos ambientes digitais. Ao continuarmos na árdua caminhada ocorrida para a realização deste trabalho, almejamos ampliar esta discussão para ambientes mais gerais, abrangentes, como é o caso da Web.

\section{Referências}

DATE, C. J. Introdução a sistemas de bancos de dados. Rio de Janeiro: Elsevier, 2003. Tradução da 8 o edição americana.

ELMASRI, R.; NAVATHE S. B. Fundamentals of database systems. 2. ed. New York: Addison Wesley, 1994.

FURTADO, A. L.; SANTOS, C. S. dos. Organização de banco de dados. 2. ed. Rio de Janeiro: Campus, 1980.

HEUSER, C. A. Projeto de banco de dados. 5. ed. Porto Alegre: Editora Sagra Luzzanato, 2004. (Série Livros Didáticos).

KORTH, H. F., SILBERSCHATZ, A. Sistema de banco de dados. 2 ed. São Paulo: Makron Books, 1995.

LITTON, G. Classificação e catalogação. São Paulo: McGraw-Hill, 1975.

MACHADO, F.; ABREU, M. Projeto de banco de dados: uma visão prática. 11. ed. São Paulo: Editora Érica, 1996.

MIRANDA, M. L. C. de. Organização e Representação do Conhecimento: fundamentos teórico-metodológico na busca e recuperação da informação em ambientes virtuais. 2005, 353 f. Tese (Doutorado em Ciência da Informação). Curso de Pós-Graduação em Ciência da Informação - Convênio CNPO/IBICT - UFRJ/ECO, Universidade Federal do Rio de Janeiro, Rio de Janeiro, 2005.

RANGANATHAN, S. R. Colon Classification. Bombay: Asia Publishing House, 1963. 126p.

RANGANATHAN, S. R. Heading and canons: comparative study of five catalogue codes. Madras: S. Viswanthan, 1955.

RANGANATHAN, S. R. Prolegomena to library classification. Bombay: Asia Publ. House, 1967.

SILVA, M. B. da; NEVES, D. A. B. A aplicação da teoria da classificação facetada em banco de dados através da modelagem conceitual. In: ENCONTRO NACIONAL DE PESQUISA EM CIÊNCIA DA INFORMAÇÃO, 11, 2011, Brasília. Anais eletrônicos... Brasília: Thesaurus, 2011. 20 p. 1 CDROM.

SILVA, M. B. da; NEVES, D. A. B. Estudo sobre o uso da teoria da classificação facetada em banco de dados. In: ENCONTRO NACIONAL DE PESQUISA EM CIÊNCIA DA INFORMAÇÃO, 11, 2010, Rio de Janeiro. Anais eletrônicos... Rio de Janeiro: CPRM - UNIRIO, 2010. 20 p. Disponível em:

<http://congresso.ibict.br/index.php/xi/enancibXI/paper/view/283>. Acesso em: 10 ago. 2014.

Recebido/Recibido/Received: 2015-08-28

Aceitado/Aceptado/Accepted: 2015-10-22 